\title{
Depressão e bem-estar subjetivo em crianças e adolescentes: teste de modelos teóricos
}

\author{
Makilim Nunes Baptista \\ Nelson Hauck Filho \\ Cassandra Cardoso \\ Universidade São Francisco, SP, Brasil
}

\begin{abstract}
Resumo
Embora sintomas de depressão e indicadores de bem-estar sejam tidos como teoricamente ortogonais, evidências sugerem, em contraste, uma correlação negativa entre eles. O presente estudo buscou testar diferentes modelos confirmatórios para explicar a relação entre sintomas depressivos e bem-estar subjetivo em crianças e adolescentes. Participaram 331 estudantes $(M=12,42$ anos; $D P=1)$, que responderam ao Inventário de Depressão Infantil (CDI), à Escala Baptista de Depressão - versão infantojuvenil (EBADEP-IJ) e à Escala de Bem-Estar Subjetivo (EBES). O melhor ajuste ocorreu para um modelo bifator, em que indicadores relacionados à depressão carregaram positivamente em um fator geral, enquanto indicadores de bem-estar subjetivo carregaram negativamente, além de se conectarem positivamente a um fator específico de aspectos positivos. Os achados integram as perspectivas aparentemente excludentes de que a depressão e o bem-estar subjetivo são ou polos opostos de um contínuo, ou entidades independentes.
\end{abstract}

Palavras-chave: Depressão; Felicidade; Análise bifator.

\section{Depression and subject well-being in children and adolescents: testing theoretical models}

\begin{abstract}
Despite symptoms of depression and indicators of subjective well-being are theoretically orthogonal entities, available evidence suggests a negative correlation between them. The present study sought to test concurrent confirmatory models with the purpose of explaining the complex relationship between indicators of depression and subjective well-being among children and adolescents. Participants were 331 students with ages from 10 to 16 years old $(M=12.42, S D=1.00)$. Participants answered the Children's Depression Inventory (CDI), the Baptista's Depression Inventory-Youth Version (EBADEP-IJ), and the Subjective Well-Being Scale (EBES). A bifactor model yielded the best fit to the data, with indicators of depression and subjective well-being loading differently (positive and negative, respectively) on a general factor, besides subjective well-being loaded as well on a separate specific positive factor. Findings shed light on the apparent controversy surrounding depression and subjective well-being as opposite poles of a same continuum or as unrelated entities.
\end{abstract}

Keywords: Depression; Happiness; Bi-factor analysis.

\section{La depressión y el bienestar subjetivo de los niños y adolescentes: la prueba de modelos teóricos}

\begin{abstract}
Resumen
Aunque los síntomas de la depresión y los indicadores de bienestar se toman como teóricamente ortogonal, la evidencia sugiere, por el contrario, una correlación negativa entre ellos. Este estudio tuvo como objetivo probar diferentes modelos confirmatorios para explicar la relación entre los síntomas depresivos y el bienestar subjetivo en los niños y adolescentes. Participado 331 estudiantes $(\mathrm{M}=12,42$ años, $\mathrm{SD}=1)$, que contestaron el Inventario de Depresión Infantil (CDI), la Escala de Depresión Baptista - versión juvenil (EBADEP-IJ) y el Bienestar Subjetivo Escala (EBE).El mejor ajuste para el modelo era bifator, en el que los indicadores relacionados con la depresión cargaran positivamente en un factor general mientras indicadores de bienestar subjetivo cargaran negativamente, y se conectan de manera positiva a un factor específico de positivos. Los resultados representan una posible integración de las perspectivas aparentemente excluyentes que la depresión y el bienestar subjetivo son o polos opuestos de un continuo, o entidades independientes.
\end{abstract}

Palabras clave: Depresión; La felicidad; El análisis bifator. 
Os quadros depressivos estão entre as doenças incapacitantes mais prevalentes no mundo (World Health Organization, 2012), afetando também as crianças e os adolescentes (Ulloa-Flores, Peña-Olvera $\&$ Nogales-Imaca, 2011). Isso faz com que se revista de importância a avaliação da sintomatologia depressiva, tanto pela possibilidade de ajudar em sua remissão parcial ou completa quanto por prevenir possíveis casos de suicídio (Bahls, 2002), especialmente em contextos de saúde pública (Sun \& Wang, 2015). De maneira geral, os sintomas depressivos centrais em crianças e adolescentes são os mesmos dos adultos, ou seja, humor triste, vazio ou irritável (afetos negativos) e alterações somáticas e cognitivas que incapacitam o funcionamento do indivíduo (American Psychiatric Association, APA, 2014; para ver algumas especificidades em adolescentes, ver Andersen \& Teicher, 2008). A presente pesquisa aborda a conexão entre sintomas de depressão e aspectos positivos do funcionamento mental em crianças e adolescentes, tais como as dimensões do bem-estar subjetivo, testando diferentes modelos confirmatórios a partir de escores no Inventário de Depressão Infantil (CDI; Kovacs, 1992), na Escala Baptista de Depressão - versão infanto-juvenil (EBADEP-IJ; Baptista, 2011) e na Escala de Bem-Estar Subjetivo (EBES; Albuquerque \& Tróccoli, 2004).

Atualmente, entende-se que a saúde mental só pode ser entendidaa partir da totalidade de seus componentes positivos e negativos (Keyes, 2002; Machado \& Bandeira, 2015;WHO, 2005). Um conceito que vem sendo estudado de forma mais sistemática na Psicologia Positiva é o bem-estar subjetivo (BES), que designa a avaliação cognitiva e afetiva que o sujeito faz de sua vida. Coerente com uma visão abrangente da saúde mental, o BES tem como domínios os afetos positivos, os afetos negativos e a satisfação com a vida (Diener, 1984; Diener, 2000; Diener, Oishi, \& Lucas, 2002). A frequência de afetos positivos e negativos vivenciados é o que determina a percepção de felicidade ou infelicidade do indivíduo (Lyubomirsky, King \& Diener, 2005), o que torna o BES um conceito útil na avaliação de quadros clínicos psicopatológicos. Uma vez que contempla aspetos positivos e negativos da saúde mental, a avaliação do BES ajuda a fornecer um mapeamento das forças e fraquezas do indivíduo em um dado momento, talvez acrescentando informação clínica única não captada pelos indicadores tradicionalmente negativos de doença mental.

Diversos achados na literatura sustentam que, de fato, transtornos mentais, especialmente a depressão, envolvem uma diminuição de aspectos positivos do BES, além da intensificação de características negativas. No caso específico da depressão, uma recente metanálise da literatura revelou um padrão consistente de correlação negativa entre aspectos positivos do funcionamento mental e sintomas da depressão, tanto em estudos transversais $(r=-.34)$ quanto em estudos longitudinais $(r=-.26$, Khazanov \& Ruscio, 2016). Outra metanálise revelou que aspectos positivos surgem enquanto um fator específico na estrutura de alguns dos principais inventários de depressão utilizados mundialmente (Shafer, 2006), o que também tem aparecido em estudos mais recentes (por exemplo, Gomez \& McLaren, 2015). Em um estudo nacional, Albuquerque e Tróccoli (2004), encontraram correlações negativas e moderadas entre $o$ fator de afetos negativos e os fatores de afetos positivos e satisfação com a vida, componentes do BES. Assim, parece haver um padrão consistente de associação negativa entre BES e indicadores de depressão quando tomado o conjunto das evidências disponíveis.

Entretanto, de um ponto de vista teórico, aspectos positivos e negativos do funcionamento mental têm sido considerados enquanto componentes independentes ou ortogonais. Diener e Emmons (1984) e Watson e Tellegen (1988) relataram que afetos positivos e negativos são distintos e, em análises fatoriais, tendem a surgir como dimensões ortogonais. De acordo com esse pressuposto de independência entre os fatores, há a possibilidade de as pessoas vivenciarem ambos esses estados internos concomitantemente, o que conduz à inferência de que a presença de sintomas de depressão não exclui necessariamente que os indivíduos possam, ao mesmo tempo, fazer uma avaliação cognitiva e afetiva positiva de suas vidas. Essa complexidade permite a existência de diferentes configurações de escores em variáveis positivas e negativas da saúde mental, trazendo uma grande diversidade de manifestações que podem ocorrer em contextos clínicos.

Uma hipótese aqui levantada para integrar o pressuposto teórico da ortogonalidade e dos achados empíricos oblíquos anteriormente relatados é um modelo bifator, já que nos estudos anteriormente citados e literatura consultada, não foram encontrados estudos com esse tipo de análise envolvendo medidas diferentes de depressão e BES. Mais especificamente, apesar de serem componentes distintos e independentes, aspectos positivos e negativos da depressão e do BES podem compartilhar uma dimensão geral de saúde/doença mental. Se essa hipótese estiver correta, a existência de um fator geral poderia explicar a correlação frequentemente encontrada entre afetos positivos e negativos, aspectos que poderiam apresentar cargas em sentido oposto nesse fator geral. Ainda, fatores específicos ortogonais a esse fator geral poderiam dar conta de uma eventual parcela de variância específica, por exemplo, dos afetos positivos que não se relaciona com (é ortogonal a) os afetos negativos. A abordagem bifator tem recebido cada vez mais atenção por permitir 
a decomposição da variância comum entre fatores que impactam todos os indicadores e fatores ortogonais secundários que impactam apenas um subconjunto desses indicadores (Reise, Moore, \& Haviland, 2010).

O presente estudo teve como objetivo investigar se um modelo bifator explica o padrão de correlações entre dimensões de instrumentos avaliativos de sintomas de depressão e de aspectos do BES em crianças e adolescentes. O foco da análise se deu em nível de escalas, não de itens especificamente, sendo testado o modelo bifator contra os modelos concorrentes de um fator e de dois fatores ortogonais, tendo escores brutos dos instrumentos como indicadores. Os instrumentos levados em consideração representam um conjunto de variáveis abrangentes tanto os aspectos positivos quanto negativos do funcionamento mental, sendo o Inventário de Depressão Infantil (CDI; Kovacs, 1992), a Escala Baptista de Depressão - versão infantojuvenil (EBADEP-IJ; Baptista, 2011) e a Escala de Bem-Estar Subjetivo (EBES; Albuquerque \& Tróccoli, 2004). O estudo se propõe a oferecer um avanço para a compreensão dos fenômenos relacionados à saúde mental de maneira ampla. O trabalho também busca fomentar uma discussão acerca do papel dos aspectos positivos enquanto de fato complementares à avaliação clínica tradicional dos transtornos mentais, já que, a inclusão de indicadores positivos do funcionamento mental na avaliação de crianças e adolescentes de países de renda baixa e média pode trazer suporte para intervenções precoces que reduzam o ônus dos transtornos (Kieling et al., 2011).

\section{Método}

\section{Participantes}

Participaram 331 sujeitos, estudantes do ensino fundamental e médio de escolas públicas e particulares de São Paulo, escolhidos por conveniência. Os participantes tinham idade entre 10 e 16 anos $(\mathrm{M}=12,42 ; \mathrm{DP}=1,00)$, sendo que $178(53,8 \%)$ eram do sexo feminino.

\section{Instrumentos}

Inventário de Depressão Infantil (CDI) (Kovacs, 1992)

O inventário é derivado do Beck Depression Inventory (BDI) e rastreia sintomas depressivos em crianças e adolescentes de sete a 17 anos de idade, sendo mundialmente utilizado e traduzido em várias línguas. O CDI é um instrumento de autorrelato tendo sido utilizada no presente estudo uma versão composta por 20 itens. Nos estudos da adaptação utilizada, escala foi considerada unifatorial, tendo sido utilizado o método de máxima verossimilhança - ML, para extração final do fator único. A variância explicada foi de 13,4\% (Gouveia et al., 1995), apesar de haver estudos com diferentes padrões componenciais, tanto no exterior (Huang \& Dong, 2014), quanto no Brasil (Coutinho, Oliveira, Pereira, \& Santana, 2014; Cruvinel, Boruchovitch, \& Santos, 2008; Golfeto, Veiga, Souza, \& Barbeira, 2002; Gouveia, Barbosa, Almeida, \& Gaião, 1995; Wathier, Dell'Aglio, \& Bandeira, 2008). Os itens apresentam sintomas afetivos, cognitivos e comportamentais. Cada item apresenta três opções de respostas, nas quais o sujeito seleciona uma. $\mathrm{O}$ valor correspondente a cada opção de resposta varia de 0 a 2 pontos, sendo o somatório dos valores das respostas o escore considerado. No que se refere à consistência interna, o alfa obtido foi 0,81 (Gouveia et al., 1995).

\section{Escala Baptista de Depressão}

(Versão Infanto-Juvenil) - EBADEP-IJ (Baptista, 2011)

A EBADEP-IJ avalia a intensidade de sintomas de depressão em crianças e adolescentes de oito a 18 anos de idade. A avaliação da sintomatologia depressiva tem como foco aspectos de tipo humor, social, irritabilidade, cognitivo, motor, vegetativo e somático. Trata-se de uma escala de autorrelato, composta inicialmentepor 45 itens e dois fatores, um constituído por itens positivos e outro por itens negativos, obtidos por meio de análise fatorial clássica e também da análise fatorial exploratória por modelagem de equação estrutural, ambos os fatores avaliam depressão como traço latente, só que invertidamente.

Os itens são pontuados em uma escala do tipo Likert de três pontos, sendo $0=$ "Nunca/Poucas Vezes", $1=$ "Algumas Vezes" e 2="Muitas Vezes/Sempre". A consistência interna das subescalas obtida no estudo original apresentou índices adequados, sendo que as medidas do alfa variaram entre 0,86 e 0,89 , enquanto o lambda 1 de Guttman variou em 0,87 e 0,91 (Baptista, 2011). A consideração de itens positivos em uma escala que rastreia sintomas depressivos é um diferencial das Escalas EBADEP, tanto na versão IJ, destinada a crianças e adolescentes, quanto na versão A, destinada a adultos. A introdução dos itens positivos amplia o campo de abrangência da escala EBADEP-IJ, trazendo maior variância explicada à escala (Baptista, 2011).

\section{Escala de Bem-Estar Subjetivo (EBES) (Albuquerque \& Tróccoli, 2004)}

A EBES mede os três principais componentes do bem-estar subjetivo, que são a satisfação com a vida, os afetos positivos e os afetos e negativos. A escala apresenta 62 itens, divididos em duas subescalas. Na primeira, 47 itens descrevem afetos positivos e negativos e na segunda, 15 itens apresentam julgamentos relativos 
à avaliação de satisfação ou insatisfação com a vida. A análise fatorial e a análise dos componentes principais revelaram três fatores referentes aos três componentes do BES. Os resultados indicaram que o fator afeto positivo é composto de 21 itens, tendo explicado $24,3 \%$ da variância. $\mathrm{O}$ fator afeto negativo é composto por 26 itens, tendo explicado $24,9 \%$ da variância. Já o fator satisfação-insatisfação com a vida é composto de 15 itens, tendo explicado $21,9 \%$ da variância. Os três fatores explicaram $44,1 \%$ da variância total do construto. Os itens são pontuados em escala Likert. $\mathrm{Na}$ primeira parte, 1 significa nem um pouco e 5 significa extremamente. Já na segunda parte, 1 significa discordo plenamente e 5 significa concordo plenamente. No estudo original, a consistência interna foi de 0,95 para o fator afeto positivo, 0,95 para afeto negativo e 0,90 para satisfação com a vida (Albuquerque \& Tróccoli, 2004).

\section{Procedimentos}

$\mathrm{O}$ projeto de pesquisa do qual deriva este artigo foi aprovado pelo Comitê de Ética da USF. Na coleta de dados, os instrumentos foram aplicados coletivamente em salas de aulas de até 30 alunos cada, após assinatura do Termo de Consentimento Livre e Esclarecido (TCLE), pelos pais ou responsáveis dos participantes. Foram aplicados a EBADEP-IJ, o CDI e a EBES. A sequência de aplicação foi alternada entre as turmas, no sentido de minimizar o efeito fadiga nos participantes. O tempo de aplicação dos instrumentos, foi de, aproximadamente, 45 minutos.

\section{Análise de Dados}

Os dados foram analisados por meio de estatísticas descritivas, para caracterização da amostra, e estatísticas inferenciais, para teste dos modelos teóricos. Para esse último propósito, foram empregadas análises confirmatórias com estimação Maximum Likelihood de tipo robusto (MLR), em função de possíveis desvios da normalidade na distribuição das variáveis. Foram testados os modelos concorrentes de um fator, dois fatores e bifator como possíveis explicações para a estrutura latente dos dados. O objetivo foi comparar o ajuste do modelo bifator hipotetizado com explicações alternativas de um fator apenas e de dois fatores oblíquos, sendo um para aspectos positivos e outro para aspectos negativos do funcionamento psicológico (vide Figura 1). O modelo de um fator parte do princípio de que sintomas de depressão e bem-estar subjetivo seriam apenas polos opostos de um mesmo contínuo, sem outros componentes adicionais a esse fator geral. Por sua vez, o modelo de dois fatores representa a hipótese de que depressão e bem-estar subjetivo são aspectos independentes, cada qual com seu próprio contínuo. Nesse caso, é possível um indivíduo experimentar sintomas de depressão, mas apresentar bem-estar subjetivo concomitantemente, ou ainda, apresentar sintomas depressivos e baixos níveis de bem-estar, sendo possíveis também outras configurações. Por fim, o modelo bifator concilia ambas as perspectivas anteriores. Ou seja, admite-se tanto a possibilidade de um fator geral (depressão e bem-estar em polos opostos) quanto de outros componentes ortogonais (Reise, Morizot, \& Hays, 2007), que, nesse caso, poderiam representar forças ou virtudes que um indivíduo poderia ter, independentemente de seu escore no fator geral de psicopatologia-saúde mental.

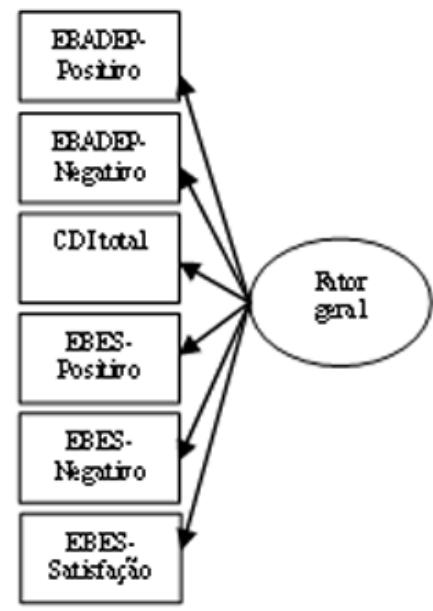

A. Modelo de umfator

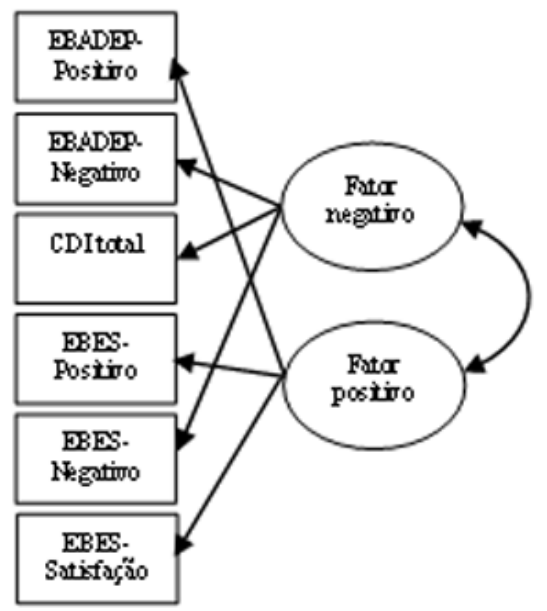

B. Modelo de doisfatores

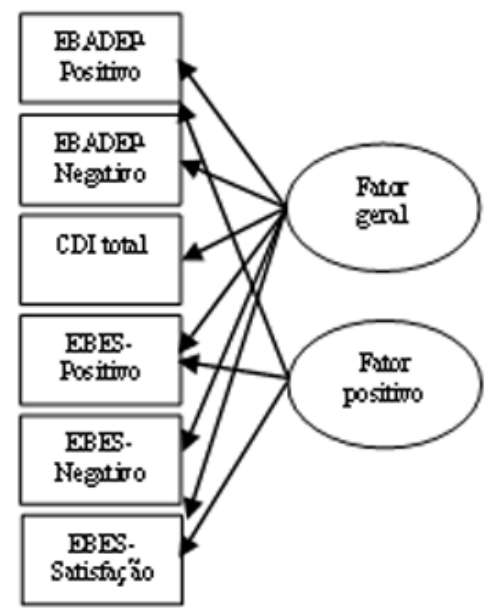

C. Modelo bif actor

Figura 1. Modelos concorrentes testados 
Para a avaliação do ajuste dos modelos, empregouse o teste qui-quadrado e os índices de ajuste Root Mean Square Error of Approximation (RMSEA $<0,08$ ). Confirmatory Fit Index $(\mathrm{CFI}>0,95)$ e Tucker-Lewis Index (TLI $>0,95)$. As análises foram conduzidas com os programas R e Mplus 7.11 (Muthén \& Muthén, 2014).

\section{Resultados}

Primeiramente, avaliaram-se os aspectos descritivos das variáveis coletadas, sendo os resultados apresentados na Tabela 1. Os valores de assimetria da EBADEP - IJ negativo e CDI Total indicam que a maior parte das pessoas obteve escores baixos. Tal inferência se confirma com a análise das médias obtidas. As médias mais baixas foram obtidas nas escalas CDI Total e EBADEP- IJ negativo. Já a média mais alta foi obtida na escala EBES Satisfação com a Vida, seguida da escala EBES negativo e EBADEP - IJ positivo.

$\mathrm{Na}$ sequência, foram feitos testes de correlação linear entre as variáveis. Os resultados são descritos na Tabela 2. As correlações variaram de fracas a fortes, sendo consistentes com as expectativas a partir da literatura. De maneira geral: 1) sintomas de depressão e afetos negativos apresentaram correlação positiva entre si; 2) aspectos positivos do BES se correlacionaram positivamente; e 3) aspectos positivos (afetos positivos e satisfação com a vida) e negativos (afetos negativos e sintomas de depressão) do funcionamento mental apresentaram correlação negativa.

Procedeu-se então ao teste dos modelos teóricos via análise fatorial confirmatória (vide Figura 1). Os resultados são apresentados na Tabela 3. Como visto, dentre os três modelos teóricos hipotetizados (um fator, dois fatores e bifator), o modelo bifator foi aquele que apresentou melhor ajuste aos dados. Ainda assim, verificou-se um qui-quadrado significativo e índices TLI e RMSEA fora do intervalo aceitável de valores, já que os três primeiros modelos testados ficaram acima de 0,10 (Tabela 3 ).

Em virtude disso, procedeu-se à inspeção dos índices de modificação, a fim de verificar possíveis relações teoricamente aceitáveis e que não haviam sido modeladas. Foram identificadas uma correlação residual (correlação positiva entre EBES-positivo e EBES-negativo) e uma carga fatorial (negativa) do CDI no fator positivo, que foram então introduzidas no modelo. Vale observar que correlações residuais entre indicadores são aquelas que ocorrem após remover a variância explicada pelos fatores do modelo, indicando a influência de outra de possível variável latente não
TABELA 1

Estatística Descritiva dos Instrumentos

\begin{tabular}{lcccccc}
\hline \multicolumn{1}{c}{ Modelo } & Média & $p$ & Mínimo & Máximo & Assimetria & Alpha \\
\hline EBADEP-IJ-pos* & 0,70 & 0,41 & 0 & 1,94 & 0,72 & 0,87 \\
EBADEP-IJ-neg & 0,50 & 0,37 & 0 & 1,65 & 1,06 & 0,91 \\
CDI total & 0,34 & 0,27 & 0 & 1,40 & 1,21 & 0,85 \\
EBES-pos & 3,24 & 0,64 & 1 & 4,71 & $-0,32$ & 0,88 \\
EBES-neg & 2,34 & 0,65 & 1,12 & 4,81 & 0,90 & 0,91 \\
EBES-sv & 3,68 & 0,71 & 1,67 & 5,00 & $-0,34$ & 0,88 \\
\hline
\end{tabular}

* $p<0,05$ para o teste Shapiro-Wilks de normalidade univaridada.

TABELA 2

Correlações Bivariadas entre as Escalas

\begin{tabular}{lccccc}
\hline \multicolumn{1}{c}{ Escalas } & 2 & 3 & 4 & 5 & 6 \\
\hline 1 EBADEP-IJ-pos & $-0,43^{*}$ & $-0,56^{*}$ & $0,48^{*}$ & $-0,38^{*}$ & $0,64^{*}$ \\
2 EBADEP-IJ-neg & & $0,76^{*}$ & $-0,17^{*}$ & $0,70^{*}$ & $-0,65^{*}$ \\
3 CDI total & & & $-0,25^{*}$ & $0,69^{*}$ & $-0,67^{*}$ \\
4 EBES-pos & & & & 0,05 & $0,35^{*}$ \\
5 EBES-neg & & & & & $-0,53^{*}$ \\
6 EBES-sv & & & & & \\
\hline
\end{tabular}

$* p<0,01$.

TABELA 3

Resultados das Análises Fatoriais Confirmatórias

\begin{tabular}{lcccccc}
\hline \multicolumn{1}{c}{ Modelo } & $\chi^{2}$ & $g l$ & $p$ & $F I$ & $T L I$ & RMSEA \\
\hline Unidimensional & 163,01 & 9 & $<0,001$ & 0,826 & 0,711 & 0,227 \\
Dois fatores & 99,10 & 8 & $<0,001$ & 0,897 & 0,808 & 0,185 \\
Bifator $^{\mathrm{a}}$ & 63,16 & 6 & $<0,001$ & 0,936 & 0,839 & 0,170 \\
Bifator $^{\mathrm{b}}$ & 4,25 & 4 & 0,373 & 1,000 & 0,999 & 0,014 \\
\hline
\end{tabular}

${ }^{a}$ Modelo bifator sem especificações adicionais.

${ }^{\mathrm{b}}$ Modelo bifator com uma correlação residual e uma carga fatorial cruzada.

especificada. O modelo bifator reespecificado, com a inclusão dessas correlações residuais, apresentou um excelente ajuste aos dados, como indicado pelo quiquadrado não significativo e pelos demais índices de ajuste. $O$ índice RMSEA, por exemplo, passou a 0,014 , sendo considerado um bom ajuste, de acordo com Hu e Bentler (1998).

No modelo bifator reespecificado, todas as variáveis apresentaram altas cargas fatoriais em um fator geral de depressão (ou psicopatologia). Os indicadores EBADEP-negativo, CDI e EBES-negativo obtiveram cargas positivas no fator geral, enquanto os indicadores EBADEP-positivo e EBES-positivo obtiveram cargas negativas no fator geral e também em um fator específico de aspectos positivos. O indicador CDI apresentou uma carga negativa de baixa magnitude (embora significativa) no fator positivo. Os resultados são apresentados na Tabela 4. 
TABELA 4

Cargas Fatoriais do Modelo Bifator Reespecificado

\begin{tabular}{lccc}
\hline \multicolumn{1}{c}{ Modelo } & $\begin{array}{c}\text { Fator } \\
\text { geral }\end{array}$ & $\begin{array}{c}\text { Fator } \\
\text { positivo }\end{array}$ & $\begin{array}{c}\text { Variância } \\
\text { residual }\end{array}$ \\
\hline EBADEP-IJ-pos & $-0,48^{*}$ & $0,78^{*}$ & 0,17 \\
EBADEP-IJ-neg & $0,89^{*}$ & & 0,21 \\
CDI total & $0,86^{*}$ & $-0,19^{*}$ & 0,23 \\
EBES-pos & $-0,20^{*}$ & $0,51^{*}$ & 0,71 \\
EBES-neg & $0,79^{*}$ & & 0,38 \\
EBES-sv & $-0,71^{*}$ & $0,38^{*}$ & 0,35 \\
\hline
\end{tabular}

$* p<0,01$.

\section{Discussão}

O presente estudo teve como proposta aprofundar o estudo da relação entre os componentes da depressão e do bem-estar subjetivo, testando uma hipótese bifator que busca integrar, de um lado, a ortogonalidade teórica e, de outro, a correlação empírica relatada para essas variáveis. A hipótese bifator foi testada contra modelos mais simples, de um fator apenas e de dois fatores ortogonais. Os modelos representam diferentes possibilidades, a saber, que depressão e bem-estar são o oposto em um mesmo contínuo (um fator), que são dois aspectos independentes (dois fatores), ou que compartilham um contínuo, mas com a possibilidade de componentes específicos (bifator).

O modelo concorrente com melhor ajuste aos dados foi o bifator, contendo um fator geral e um fator específico. O fator geral foi aqui interpretado como uma dimensão ampla de psicopatologia-saúde mental, captando os aspectos centrais de afetos negativos, humor deprimido e alterações cognitivas características da depressão (Huang \& Dong, 2014). No DSM-5 (APA, 2014), boa parte dos transtornos mentais (incluindo a depressão) apresentam como fator de risco o fator Neuroticismo, de modo que eventos negativos de vida podem ser um gatilho para o desenvolvimento de diversos transtornos mentais nesse caso. De maneira análoga, ao avaliarem a estrutura conjunta de diversos transtornos mentais, Caspi et al. (2014) relataram a existência de um fator geral - chamado de fator " $p$ " -, que se caracteriza por uma propensão ao desenvolvimento de todas as psicopatologias. Uma hipótese aqui lançada, portanto, é que o fator geral encontrado é um componente responsável pelo desencadeamento de transtornos mentais, especialmente a depressão. Essa afirmação, não obstante, precisa ser consubstanciada por outras investigações empíricas. De todo modo, outras investigações também encontraram uma estrutura bifator ao investigarem indicadores de psicopatologia infantil e juvenil em geral (sintomas internalizantes e externalizantes), como o Strengths and Difficulties Questionnaire (Caci, Morin, \& Tran, 2015; Kóbor, Takács, \& Urbán, 2013).

Em contraste, o fator específico do modelo bifator parece agregar informação relacionada a afetos positivos e felicidade (Diener, Oishi, \& Lucas, 2002). Dada a ortogonalidade desse fator com relação ao fator geral descrito anteriormente, os achados indicam que uma parte desses componentes positivos da saúde mental é, de fato, independente dos tradicionais indicadores de transtornos. Em outras palavras, o modelo bifator integra as perspectivas contrastantes de que a) afetos positivos e negativos são ortogonais e que b) essas variáveis são oblíquas. Apesar de o fator geral do modelo ser coerente com uma visão unidimensional da psicopatologia e da saúde mental, o fator específico encontrado também sugere a existência de forças ou virtudes que um indivíduo pode ter mesmo quando apresenta algum transtorno como a depressão. Embora, teoricamente, a depressão e o bem-estar compartilhem uma dimensão (na qual se localizam em polos opostos), o fato de um indivíduo ter sintomas de depressão não excluiria a possibilidade de ele também apresentar características positivas, tais como afetos positivos e satisfação com a vida - pelo menos em alguma medida. Os achados são coerentes com aqueles relatados por outras investigações no contexto da depressão, como a metanálise de Shafer (2016), que encontrou um fator positivo em alguns dos principais inventários de depressão, e com o estudo de Gomez e McLaren (2015), que relatou, para a escala de depressão CES-D, uma estrutura similar à aqui relatada.

Em estudos futuros, uma perspectiva de discriminar ambos os fatores geral e específico aqui relatados seria a partir do estabelecimento de correlações diferenciais com variáveis externas para cada um desses fatores. Um teste pertinente nesse sentido seria incluir no modelo variáveis de desfecho, por exemplo, tentativa de suicídio, e avaliar o quanto cada um desses fatores explica tal fenômeno. Nesse caso, além da contribuição de cada fator, dada a ortogonalidade entre eles, seria de importância teórica e clínica investigar se ambos os fatores interagem na explicação do desfecho. Ou seja, pode ser possível que não apenas o fator geral de psicopatologia-saúde mental $(+)$ e o fator específico positivo $(-)$ se relacionem ao desfecho, mas também que o fato de um indivíduo pontuar alto no fator positivo pode exercer um efeito protetivo, amenizando o efeito do fator geral e, dessa maneira, diminuindo a probabilidade de ocorrer uma tentativa de suicídio subsequente. Investigações dessa natureza poderão oferecer uma 
informação valiosa ao profissional clínico, uma vez que identificam características específicas nas quais intervir na prevenção do desfecho. Uma implicação dos resultados da presente investigação é que mesmo que indicadores de transtornos mentais se sobreponham, em parte, a indicadores de aspectos positivos da saúde mental, essa sobreposição não é perfeita: afetos positivos e satisfação com a vida apresentam, de fato, um componente único independente. Assim, a inclusão de indicadores positivos do funcionamento mental, nas avaliações clínicas, acrescenta informação não contida nos tradicionais inventários de depressão e de outros transtornos. Nesse sentido, a EBADEP-IJ se mostra um instrumento vantajoso por conter um fator que contempla, especificamente, tais indicadores.

É importante destacar que as reespecificações feitas no modelo não alteram as hipóteses aqui esboçadas. Como os três instrumentos empregados apresentam muitos itens com conteúdo similar, não surpreende a existência de correlações residuais, algo que ocorreu entre os indicadores EBES-positivo e EBES-negativo. Além disso, o instrumento CDI, apesar de não conter uma escala específica de aspectos positivos (como ocorre com a EBADEP-IJ), revelou ser informativo tanto acerca do fator geral, quanto do fator específico, ainda que em menor medida, fator no qual apresentou carga cruzada negativa. Vale ressaltar, a esse respeito, que as duas fontes de variância estão misturadas nos itens do CDI, que são todos negativos, mas estão mais claramente separadas nos itens da EBADEP-IJ, que possui uma escala específica para aspectos positivos.
Por último, vale mencionar que existe uma interpretação alternativa para os resultados, especificamente no que se refere ao fator específico positivo encontrado. Como relatado em outras investigações (e.g., Ray, Frick, Thornton, Steinberg, \& Cauffman, 2015), itens com escore reverso podem produzir fatores de método. Um fator de método tende a representar o efeito específico da maneira de pontuar o item, em detrimento do conteúdo desse item (Geiser \& Lockhart, 2012). Em virtude disso, não é possível descartar aqui a possibilidade de que o fator específico positivo constitui apenas o efeito da construção das sentenças dos itens, introduzindo um viés nos escores dos indivíduos. Novamente, estudos acrescentando covariáveis ao modelo serão fundamentais para testar essa possibilidade.

O presente estudo apresenta algumas limitações. Em primeiro lugar, não foi o foco do presente controlar um possível efeito da desejabilidade social dos itens de autorrelato dos instrumentos analisados. Estudos recentes têm sugerido que, quando presente no enunciado dos itens, o componente de desejabilidade social tende a enviesar as cargas do fator geral na análise fatorial (Bäckström, Björklund, \& Larsson, 2009; Bäckström \& Björklund, 2014). Seria necessário, em função disso, empreender novos estudos buscando controlar essa possível influência, a fim de estabelecer com mais clareza de que maneira cada indicador no modelo se relaciona ao fator geral quando controlado esse viés. Diversificar o tipo de indicadores também poderia auxiliar, por exemplo, incluindo heterorrelato ou ainda variáveis relacionadas a marcadores biológicos.

\section{Referências}

Albuquerque, A. S. \& Tróccoli, B. T. (2004). Desenvolvimento de Uma Escala de Bem-Estar Subjetivo. Psicologia: Teoria e Pesquisa, 20(2), 153-164. http://dx.doi.org/10.1590/s0102-37722004000200008

American Psychiatric Association (APA). (2014). Manual diagnóstico e estatístico de transtornos mentais: DSM ( $5^{\mathrm{a}}$ ed.). Porto Alegre: Artmed.

Andersen, S. L. \& Teicher, M. H. (2008). Stress, sensitive periods and maturational events in adolescent depression. Trends in Neurosciences, 31(4), 183-191. http://dx.doi.org/10.1016/j.tins.2008.01.004

Bäckström, M. \& Björklund, F. (2014). Social desirability in personality inventories: The nature of the evaluative factor. Journal of Individual Differences, 35(3), 144-157. http://dx.doi.org/10.1027/1614-0001/a000138

Bäckström, M., Björklund, F., \& Larsson, M. R. (2009). Five-factor inventories have a major general factor related to social desirability which can be reduced by framing items neutrally. Journal of Research in Personality, 43(3), 335-344. doi:10.1016/j.jrp.2008.12.013 http://dx.doi.org/10.1016/j.jrp.2008.12.013

Bahls S. C. (2002). Aspectos clínicos da depressão em crianças e adolescentes. Jornal de Pediatria, 78(5), 359-366.

Baptista, M. N. (2011). Escala Baptista de Depressão - Versão Infanto-Juvenil (EBADEP-IJ). (Manual nãopublicado). Programa de Pós-Graduação Stricto Sensu em Psicologia, Universidade São Francisco, Itatiba. São Paulo. Brasil.

Caci, H., Morin, A. J. S., \& Tran, A. (2015). Investigation of a bifator model of the Strengths and Difficulties Questionnaire. European Child \& Adolescent Psychiatry, 24(10), 1291-301. http://dx.doi.org/10.1007/s00787-015-0679-3 
Caspi, A., Houts, R. M., Belsky, D. W., Goldman-Mellor, S. J., Harrington, H., Israel, S., ..., \& Moffitt, T. E. (2014). The p Factor: One General Psychopathology Factor in the Structure of Psychiatric Disorders? Clinical Psychological Science: A Journal of the Association for Psychological Science, 2(2), 119-137. http://dx.doi. org/10.1177/2167702613497473

Coutinho, M. P. L., Oliveira, M. X., Pereira, D. R., \& Santana, I. O. (2014). Indicadores psicométricos do Inventário de Depressão Infantil em amostra infanto-juvenil. Avaliação Psicológica, 13(2), 269-276.

Cruvinel, M., Boruchovitc, E., \& Santos, A. A. A. dos. (2008). Inventário de Depressão Infantil (CDI): Análise dos parâmetros psicométricos. Fractal: Revista de Psicologia, 20(2), 473-490. http://dx.doi.org/10.1590/s198402922008000200013

Costello, E. J., Erkanli, A., \& Angold, A. (2006). Is there an epidemic of child or adolescent depression? Journal of Child Psychology and Psychiatry, 47(12), 1263-1271. http://dx.doi.org/10.1111/j.1469-7610.2006.01682.x

Diener, E. (1984). Subjective well-being. Psychological Bulletin, 95, 542-575. http://dx.doi.org/10.1037/00332909.95.3.542

Diener, E., \& Emmons, R. A. (1984).The independence of positive and negative affect. Journal of Personality and Social Psychology, 47(5),1105-1117. http://dx.doi.org/10.1037/0022-3514.47.5.1105

Diener, E. (2000). Subjective well-being: the science of happiness and a proposal for a national index. American Psychologist, 55(1), 34-43. http://dx.doi.org/10.1037/0003-066X.55.1.34

Diener, E., Oishi, S., \& Lucas, R. E. (2002). Subjective well-being: The Science of Happiness and Life Satisfaction. In N. C. R. Snyder \& S. J. Lopez (Eds.), Handbook of positive psychology (pp. 63-73). New York: Oxford University Press.

Geiser, C., \& Lockhart, G. (2012). A comparison of four approaches to account for method effects in latent state-trait analyses. Psychological Methods, 17(2), 255-83. http://dx.doi.org/10.1037/a0026977

Golfeto, J. H., Veiga, M. H.; Souza, L de., \& Barbeira, C. (2002). Propriedades psicométricas do Inventário da Depressão Infantil (CDI) aplicado em uma amostra de escolares de Ribeirão Preto. Revista de Psiquiatria Clínica, 29(2), 66-70.

Gomez, R., \& McLaren, S. (2015). The Center for Epidemiologic Studies Depression Scale: Support for a Bifator Model With a Dominant General Factor and a Specific Factor for Positive Affect. Assessment, 22(3), 351-360. http://dx.doi.org/10.1177/1073191114545357

Gouveia, V. V., Barbosa, G. A., Almeida, H J F de., Gaião, A de A (1995). Inventário de Depressao infantil - CDI: estudo de adaptação com escolares de João Pessoa. Jornal Brasileiro de Psiquiatria, 44(7), 345-349.

Hu, L. T. \& Bentler, P. M. (1998). Fit indices in covariance structure modeling: Sensitivity to underparameterized model misspecification. Psychological Methods, 3, 424-453. http://dx.doi.org/10.1037/1082-989X.3.4.424

Huang, C. \& Dong, N. (2014). Dimensionality of the Children's Depression Inventory: Meta-analysis of Pattern Matrices. Journal Child Family Studies, 23, 1182-1192. http://dx.doi.org/10.1007/s10826-013-9779-1

Keyes, C. L. M., Shmotkin, D., \& Ryff, C. D. (2002). Optimizing well-being: The empirical encounter of two traditions. Journal of Personality and Social Psychology, 82(6), 1007-1022. http://dx.doi.org/10.1037/0022-3514.82.6.1007

Khazanov, G. K. \& Ruscio, A. M. (2016). Is low positive emotionality a specific risk factor for depression? A meta-analysis of longitudinal studies. Psychological Bulletin, 142(9), 991-1015. http://dx.doi.org/10.1037/bul0000059

Kieling, C., Baker-Henningham, H., Belfer, M., Conti, G., Ertem, I., Omigbodun, O., Rohde, L.A., Srinath, S., Ulkuer, N., \& Rahman, A. (2011). Child and adolescent mental health worldwide: evidence for action. The Lancet, 378, 1515-1525. http://dx.doi.org/10.1016/S0140-6736(11)60827-1

Kóbor, A., Takács, Á., \& Urbán, R. (2013). The Bifator Model of the Strengths and Difficulties Questionnaire. European Journal of Psychological Assessment, 29(4), 299-307. http://dx.doi.org/10.1027/1015-5759/a000160

Kovacs, M. (1992). Children Depression Inventory - CDI: Manual. New York: Multi Health Systems.

Lyubomirsky, S., King, L., \& Diener, E. (2005) The Benefits of Frequent Positive Affect: Does Happiness Lead to Success? Psychological Bulletin, 131(6), 803-855. http://dx.doi.org/10.1037/0033-2909.131.6.803

Machado, W. L. \& Bandeira, D. R. (2015). Positive Mental Health Scale: Validation of the Mental Health Continuum - Short Form. Psico-USF, Bragança Paulista, 20(2), 259-274. http://dx.doi.org/10.1590/1413-82712015200207

Muthén, L. K. \& Muthén, B.O. (2014). Mplus user's guide. (7ํeㄹ.). Los Angeles: Muthén \& Muthén.

Radloff, L. S. (1977). The CES-D scale: A self-report depression scale for research in the general population. Applied Psychological Measurement, 1, 385-401. http://dx.doi.org/10.1177/014662167700100306

Ray, J. V., Frick, P. J., Thornton, L. C., Steinberg, L., \& Cauffman, E. (2015). Positive and Negative Item Wording and Its Influence on the Assessment of Callous-Unemotional Traits. Psychological Assessment, sem paginação específica. http://dx.doi.org/10.1037/pas0000183

Reise, S. P., Morizot, J., \& Hays, R. D. (2007). The role of the bifator model in resolving dimensionality issues in health outcomes measures. Quality of Life Research, 16(Suppl 1), 19-31. http://dx.doi.org/10.1007/s11136-007-9183-7

Reise, S. P., Moore, T. M., \& Haviland, M. (2010). Bifator Models and Rotations: Exploring the Extent to Which Multidimensional Data Yield Univocal Scale Scores. Journal of Personality Assessment, 92(6), 544-559. http://dx.doi.org/10.1080/00223891.2010.496477 
Seligman, M. E. P., Steen, T. Park, N., \& Peterson, C. (2005).Positive Psychology Progress.Empirical Validation of Interventions. American Psychologist, 60(5), 410-421. http://dx.doi.org/10.1037/0003-066X.60.5.410

Shafer, A. B. (2006). Meta-analysis of the factor structures of four depression questionnaires: Beck, CES-D, Hamilton, and Zung. Journal of Clinical Psychology, 62(1), 123-146. http://dx.doi.org/10.1002/jclp.20213

Sun, S., \& Wang, S. (2015). The Children's Depression Inventory in Worldwide Child Development Research: A Reliability Generalization Study. Journal of child and family studies, 24, 2352-2363. http://dx.doi.org/10.1007/ s10826-014-0038-x

Ulloa-Flores, R. E., Peña-Olvera, F., \& Nogales-Imaca, I. (2011). Tratamiento multimodal de niños y adolescentes com depresión. Salud Mental, 34, 403-407.

Watson, D., Clark, L. A., \& Tellegen, A. (1988). Development and validation of brief measures of positive and negative affect: The PANAS Scales. Journal of Personality and Social Psychology, 54(6), 1063-1070. http://dx.doi. org/10.1037/0022-3514.54.6.1063

Wathier, J. L., Dell'Aglio, D. D., \& Bandeira, R, D. (2008). Análise fatorial do Inventário de Depressão Infantil (CDI) em amostra de jovens brasileiros. Avaliação Psicológica, 7(1), 75-84.

World Health Organization (WHO). (2005). Promoting mental health: Concepts, emerging evidence, practice. Geneva.

World Health Organization (WHO). (2012). Depression. A Global Public Health Concern. Department of Mental Health and Substance Abuse. Recuperado de: http://who.int/mental health/management/depression/who paper depression wfmh_2012.pdf

Autores:

Makilim Nunes Baptista - Doutor, Universidade São Francisco - USF.

Nelson Hauck Filho - Doutor, Universidade São Francisco - USF.

Cassandra Cardoso - Doutoranda, Universidade São Francisco - USF. Universidade Regional Integrada do Alto Uruguai e das Missões (URI - Campus de Erechim-RS)

\section{Endereço para correspondência:}

Nelson Hauck Filho

Rua Waldemar César da Silveira, 105 - Vl. Cura D’Ars (SWIFT)

13045-51 Campinas, SP, Brasil

<hauck.nf@gmail.com>

Recebido em: 03.02.2016

Aceito em: 21.09.2016 